QCD Evolution Workshop: From Collinear to Non-Collinear Case International Journal of Modern Physics: Conference Series Vol. 4 (2011) 179-189

(C) World Scientific Publishing Company

DOI: $10.1142 / \mathrm{S} 2010194511001681$

\title{
PARTONIC INTERPRETATION OF GENERALIZED PARTON DISTRIBUTIONS
}

\author{
G. R. GOLDSTEIN \\ Department of Physics and Astronomy, Tufts University \\ Medford, MA 02155, USA \\ gary.goldstein@tufts.edu \\ SIMONETTA LIUTI \\ Department of Physics, University of Virginia \\ Charlottesville, VA 22901, USA \\ sl4y@virginia.edu
}

\begin{abstract}
Dispersion relations have been applied to hard exclusive processes suggesting that generalized parton distributions can be fully determined from their values on a kinematical ridge. We show significant pitfalls associated that vitiate this approach. We suggest an improved treatment of this assumption. Secondly, in order for a partonic interpretation to hold throughout the accessible kinematic region, semi-disconnected diagrams should not contribute. We show, however, that this condition is not satisfied for non-forward kinematics at leading order. Lastly we present our recent flexible parameterization of GPDs and indicate its application to neutral pseudoscalar electroproduction.
\end{abstract}

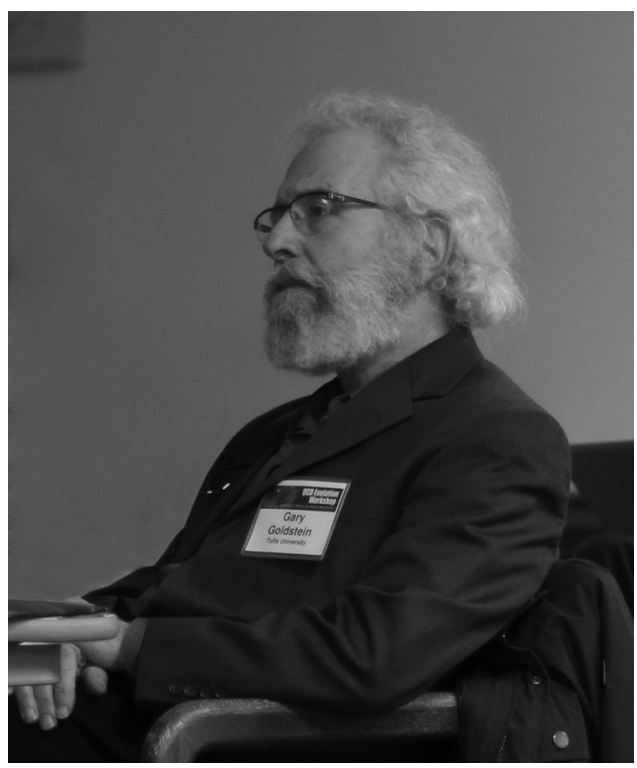




\section{Thresholds and Dispersion Relations for GPDs}

Dispersion Relations (DRs) have been advocated to facilitate the extraction from deeply virtual exclusive experiments, such as Deeply Virtual Compton Scattering (DVCS), of the soft matrix elements for hard exclusive processes, the Generalized Parton Distributions (GPDs), and to determine their model parameters ${ }^{1,2,3}$. We have shown that there are important limitations to the use of DRs for processes described by GPDs [4].

DRs have a long history in hadronic physics. For a general exclusive, two body hadronic reaction, invariant amplitudes can be written in terms of energy and angle variables. such as the Mandelstam variables $s$ and $t$, or $\nu=(s-u) / 4 M$ and $t$. When the energy variable $\nu$ is continued into the complex plane, the amplitudes become analytic functions over regions of the complex plane. Unitarity of the amplitudes determines the physical intermediate states that, in turn determine branch cuts in the complex energy plane. Each physical state has a kinematic threshold that fixes the branch point. DRs were derived for inclusive Deep Inelastic Scattering (DIS) as well, when viewed as forward virtual Compton scattering ${ }^{5}$. DIS can be considered as a special case of elastic scattering where unitarity relates the imaginary part of the forward amplitude to the total cross section, the inclusive sum over all physical final states allowed by the energy.

A connection with the partonic structure, through the Operator Product Expansion, therefore QCD, can be established e.g. by following the derivation in Ref. 5, where the important assumption is made that the support for both the integrals defining the Mellin moments of the operators and the final amplitude is $x_{B j} \in[-1,1]$, in the asymptotic limit, $Q^{2} \rightarrow \infty$. More specifically, two steps are taken in establishing DRs for DIS: $i$ ) the identification of the physical threshold for the scattering process, $\nu_{t h}$, with the continuum threshold, $\nu_{C}=M+m_{\pi}$, or $x_{C}=1$, appearing in the integral definition of the scattering amplitude; ii) the identification of $x_{B j}$ with the partonic variable present in the factorized amplitude.

We have shown that these assumptions cannot be carried straightforwardly to the off-forward case described e.g. in DVCS. In fact, one has a mismatch between the supports for the scattering amplitude and for the corresponding DR, namely $\nu_{C} \neq \nu_{t h}$. The mismatch exists for both $\nu$ and $\zeta$. This point does not touch upon the partonic aspects of the process. However, in the factorized form of DVCS, described by a handbag picture, $x_{B j}$ in $\left.i i\right)$ is replaced by two longitudinal fractions, $X$ and $\zeta$, where $X \equiv(k q) /(P q)$ and the external variable, the skewness, $\zeta=(q \Delta) /(q P) \approx$ $Q^{2} /\left(2 M \nu_{L a b}\right), \Delta$ being the momentum transfer for the two body scattering process, $\Delta^{2}=t$ (see Ref. 6, 7 for reviews). The expression for the DVCS amplitude at leading order in QCD factorization is

$$
T^{\mu \nu}\left(\nu, Q^{2}, t\right)=-\frac{1}{2} g_{T}^{\mu \nu} \bar{u}\left(p^{\prime}\right) \hat{n} u(p) \sum_{\text {flavors }} e_{f}^{2} \mathcal{H}_{f}(\xi, t),
$$


where the analog of the Compton Form Factor (CFF) is

$$
\mathcal{H}_{f}^{+}(\xi, t)=\int_{-1}^{+1} d x \frac{H_{f}^{+}(x, \xi, t)}{x-\xi+i \epsilon} .
$$

The GPD $H(x, \xi, t)$ is convoluted with the hard part, $1 /(x-\xi+i \epsilon)$, and integrated over $x$ in the range $[-1,1]$. Crossing symmetry is implemented by

$$
H_{f}^{( \pm)}(x, \xi, t)=H_{f}(x, \xi, t) \mp H_{f}(-x, \xi, t),
$$

recalling that for PDFs, $q(-x)=-\bar{q}(x)$ relates negative $x$ to positive $x$ antiquark probability.

It follows from Eq. (2) that $\operatorname{Im} \mathcal{H}(\xi, t)=H(\xi, \xi, t)$. To relate this to the discontinuity across the physical branch cut of a holomorphic function, unitarity is invoked through the insertion of a complete set of intermediate states. The resulting analytic structure allows the DR to be written,

$$
\pi \operatorname{Re} \mathcal{H}^{( \pm)}(\xi, t)=P . V . \int_{-1}^{\xi_{t h}} d x \frac{H^{( \pm)}(x, x, t)}{x-\xi}+\int_{\xi_{t h}}^{+1} d x \frac{H_{u n p h y s}^{( \pm)}(x, x, t)}{x-\xi}
$$

where the subscript unphys emphasizes that the integration should be over the whole range, but because the integration variable is now interpreted as the skewness, "external" to the quark loop, a threshold mismatch ensues due to the inelasticity of the two body process for non-zero $t$. In fact $\zeta_{t h}=\left[-t+\left(t^{2}-4 M^{2} t\right)^{1 / 2}\right] / 2 M$ for $Q^{2}>>t$, the physical threshold for the two body, $\gamma^{*} p \rightarrow \gamma p^{\prime}$ scattering process, originates from the limiting values for the angles defining the invariant $t=\left(q-q^{\prime}\right)^{2}$. One obtains in the limit $Q^{2}>>t, t_{\text {min }}=Q^{4} / 4 s-\left(q^{C M}-q^{\prime C M}\right)^{2}=-M^{2} \zeta^{2} /(1-\zeta)$.

In DVCS the region $x \in\left[\xi_{t h}, 1\right]$ is unphysical and the second term in Eq.(4) cannot be obtained from experiment. The physical meaning of this discrepancy is illustrated in left Fig.1 where both the continuum and physical thresholds for several variables describing DVCS, $s, \nu$ and $\zeta$, are plotted as a function of $t$. For $s$, as $Q^{2}$ increases, only higher and higher invariant mass states are sampled. The threshold mismatch seen in these fixed $t$ DRs in $\zeta$ could be reduced by introducing new variables, a method used in hadronic processes. We have shown the consequences of introducing a jet mass in the factorized picture ${ }^{8}$. It is this point about the sufficiency of the "ridge" that we examined with care and concluded that it is not sufficient.

We considered examples of models that should satisfy DRs. Particularly, the quark diquark model ${ }^{3}$ ), being covariant, satisfies the polynomiality condition thus allowing the GPD to be continued into the large $\xi$ or small $x / \xi$ region in which the analyticity requirements apply ${ }^{1}$. The subtraction, $\Delta(\xi, t)$ the difference between the evaluation of Eq.(2) and Eq.(4) for the symmetric case is presented in right Fig.1, which clearly displays non negligible $\xi$ and $t$ variations of $\Delta(\xi, t)$, thus demonstrating that $\Delta(\xi, t)$ cannot be identified with a dispersion subtraction constant. Given that our subtraction "constant" $\Delta(\zeta, t)$ is actually a function of $\zeta$, due to the threshold dependence, there cannot be a direct relation to either the "D-term" [1] or the $J=0$ fixed pole [9], although at high $|t|$ and $Q^{2}$ there is a flattening out. 

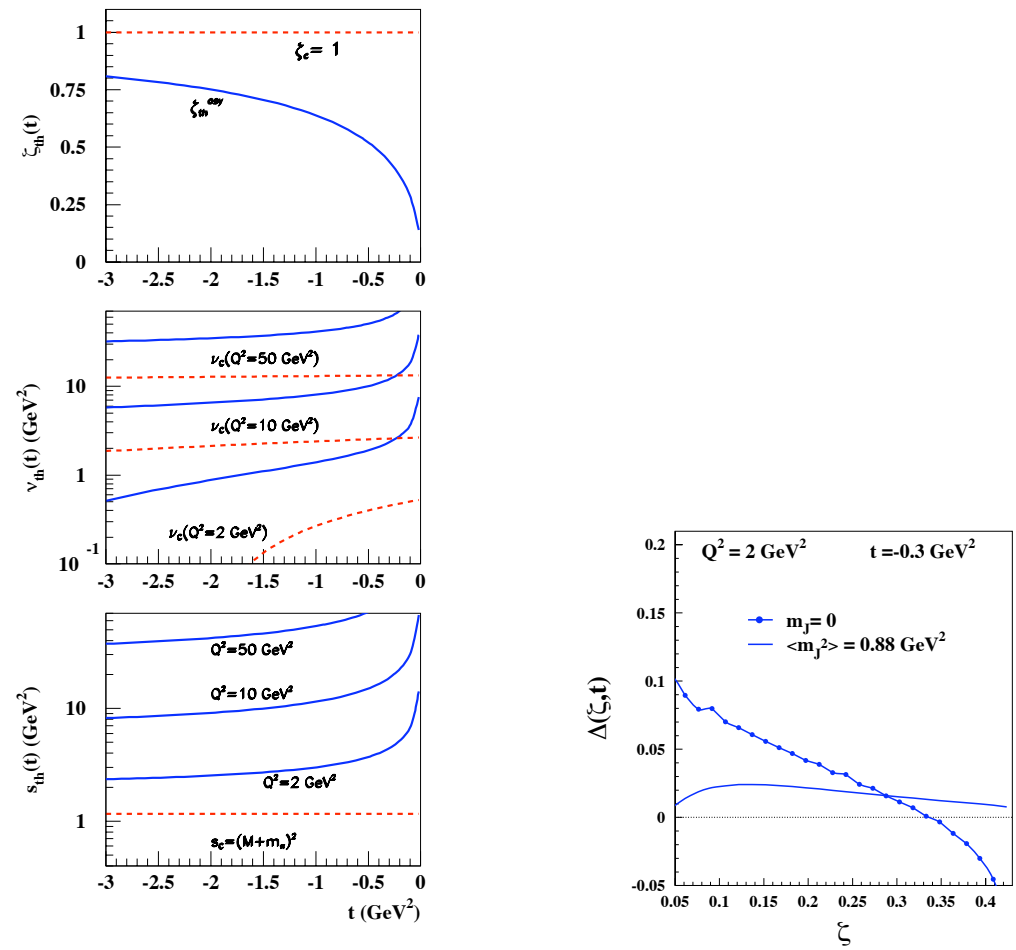

Fig. 1. Left: Thresholds for the variables $\zeta, \nu$ and $s$ plotted vs. $t$. The dashed lines are the continuum thresholds whereas the full lines represent the physical thresholds (see text). Right: Difference between the dispersion relation and direct calculation in a scalar quark-diquark model including the hard jet hadronic mass as from Eqs. (5) and (6).

Is there a way to restore a sensible picture of partonic intermediate states? The prescription we suggested as an alternative to analytic continuation aims at reducing the kinematical threshold mismatch by allowing a range of spectator masses, $m_{J}$, for the hard partonic jet. Following Ref. 8, 10, hard propagators for the struck quark in the hard part of the handbag with a variable jet mass replace

$$
\frac{1}{\zeta-X+i \epsilon} \text { with } \frac{1}{\zeta\left(1+\frac{m_{J}^{2}}{Q^{2}}\right)-X+i \epsilon} .
$$

The dispersion relation becomes

$$
\operatorname{Re} H=P V \int d X \int d m_{J}^{2} \rho\left(m_{J}^{2}\right) \frac{H\left(X,\left(1+\frac{m_{J}^{2}}{Q^{2}}\right) X, t\right)}{\zeta-X},
$$

where $\rho\left(m_{J}^{2}\right)$ is a jet mass distribution. The results (see Ref. 4) show that the new set of variables take better account for the correct range of integration over the partons' virtuality and transverse momentum. 
Concluding this section, we have shown the limitations of applying DRs to deeply virtual exclusive processes. To pin down GPDs we advocate comprehensive measurements of both the real and imaginary components through various asymmetries and cross section components in a wide range of all kinematical variables, $\zeta, t$ and $Q^{2}$.

\section{The ERBL Region and Partons in GPDs}

Because the momenta of the outgoing and incoming quark and proton are different in GPDs, two distinct kinematical regions can be defined. By denoting $X=k^{+} / P^{+}$ the light cone momentum fraction of the struck quark relative to the initial proton momentum, $P^{+}$, and by $X-\zeta=k^{+} / P^{+}$the corresponding momentum fraction of the returning quark $\left(\zeta=\Delta^{+} / P^{+}\right.$represents the t-channel momentum transfer fraction), two distinct regions appear. In the $X>\zeta$ region both the struck and returning quark carry positive momentum fractions of the initial proton momentum. This is called the DGLAP region because of the way parton evolution is expected to proceed. The $X<\zeta$ region has been interpreted as describing a quark-antiquark pair emerging from the proton, more similar to the generalization of a distribution amplitude. Evolution proceeds through the Efremov-Radyushkin-Brodsky-Lepage (ERBL) mechanism. This way of interpreting the ERBL region was proposed at the inception of DVCS studies. In Ref. 4 we found a reason of concern in noticing that it imposes several seemingly artificial constraints for partonic based descriptions and model building. The most compelling issue examined here is whether the ERBL region relates at all to the proton's partonic substructure or whether the quark anti-quark pair emerges as a vacuum hadronic fluctuation.

Three conditions are essential to characterize a partonic description ${ }^{5}$ :

(1) the support in $X$ is defined by the region $|X| \leq 1$;

(2) analytic properties of the partonic amplitude have to correspond to the emission and absorption of quarks/antiquarks via well defined on-mass shell intermediate hadronic states;

(3) the quark-proton vertices have to be connected.

A careful derivation of the parton model from the connected matrix elements for the non-local quark and gluon fields operators that enter inclusive hard processes was given in Ref. 5 (a formal extension to the off-forward case was given in Ref. 11). There it was pointed out that a "simple" physical picture does not emerge uniquely and naturally from the structure of the correlator, but that analytic properties need to be taken into consideration. We extended the arguments of Ref. 5 to the ERBL region that similarly presents a more complicated partonic structure. A factorized form was derived in Ref. 12 for the DVCS amplitude as

$$
\begin{aligned}
\mathcal{F} & =\int_{-\zeta+1}^{1} d X\left(\frac{1}{X-\zeta+i \epsilon}-\frac{1}{X+i \epsilon}\right) \\
& \times \int d z^{-} e^{i q^{+} z^{-}}\left\langle P^{\prime}\left|\bar{\psi}\left(z^{-}\right) \gamma^{+} \psi(0)\right| P\right\rangle .
\end{aligned}
$$


The matrix element in the equation corresponds to GPDs defined e.g. in the unpolarized case as

$$
\begin{array}{r}
\int d z^{-} e^{i q^{+} z-}\left\langle P^{\prime}\left|\bar{\psi}\left(z^{-}\right) \gamma^{+} \psi(0)\right| P\right\rangle= \\
\bar{U}\left(P^{\prime}\right)\left[H(X, \zeta, t) \gamma^{+}+E(X, \zeta, t) \frac{-i \sigma^{+, \lambda}}{2 M} \Delta_{\lambda}\right] U(P)
\end{array}
$$

To clarify the identification of partonic or non-partonic interpretations of the GPDs we can explicitly expand the quark field operators of Eq.(8) in terms of light front free field variables (implicitly employing the operator product expansion as in Ref. 5). We use the decomposition into creation and annihilation operators focusing for simplicity on $H$, as in Ref. 7. The relation to possible on-shell intermediate states can be explored by inserting a complete set between the quark field operators acting between the incoming or outgoing proton states. Whether or not the corresponding diagrams contribute depends on the values of $X$ and $\zeta$ and the momenta. It was pointed out by $\mathrm{Jaffe}^{5}$ that even in the forward case, when $\zeta=0$ and $X<0$ there are semi-disconnected contributions to the "unitarity diagrams", shown in Fig. 2, that do not have a partonic interpretation. However, there are two equivalent forms of the product of the two non-local interacting quark field operators, using the anticommutation of the operators on the null-plane As a result, from the equivalence of these two forms on can deduce an equivalence between the non-partonic/semidisconnected diagrams with $X<0$ for quarks and, through the alternative ordering of the fields, a partonic distribution for anti-quarks with $X>0$ represented by the usual connected configuration. The important question here is whether or not this kind of equivalence can be established in the off-forward case in order to allow a partonic interpretation of GPDs.

To consider these questions, insert intermediate states in Eq.(8) using completeness, and associate each vertex with plus momentum conservation. In the forward limiting case one obtains

$$
H(X, 0,0)=\sum_{n} \delta\left(P^{+}-X P^{+}-P_{n}^{+}\right)|\langle n|\psi| P\rangle|^{2}
$$

so for quarks with $X>0$ this corresponds to the usual parton picture in Fig. 2a. For $X<0$ the delta function requires that the intermediate state momentum exceeds the proton $p^{+}$, that is $p_{n}^{+}=P^{+}+|X| p^{+}$. That condition can be satisfied by the creation of a pair as in Fig. 2b, as well as through annihilation of a pair, as shown in detail by $\mathrm{Jaffe}^{5}$.

Similarly, in the off-forward case

$$
H(X, \zeta, t)=\sum_{n} \delta\left(P^{+}-X P^{+}-P_{n}^{+}\right)\left\langle P^{\prime}|\bar{\psi}| n\right\rangle\langle n|\psi| P\rangle
$$

By expanding $\psi$ and concentrating on one term to illustrate the procedure, we have

$$
\begin{aligned}
& \left\langle P^{\prime}\left|d_{\lambda}\left(X-\zeta,-\boldsymbol{k}_{T}^{\prime}\right)\right| n\right\rangle\left\langle n\left|b_{-\lambda}\left(X, \boldsymbol{k}_{T}\right)\right| P\right\rangle \\
& \times \delta\left(P^{+}-X P^{+}-P_{n}^{+}\right),
\end{aligned}
$$



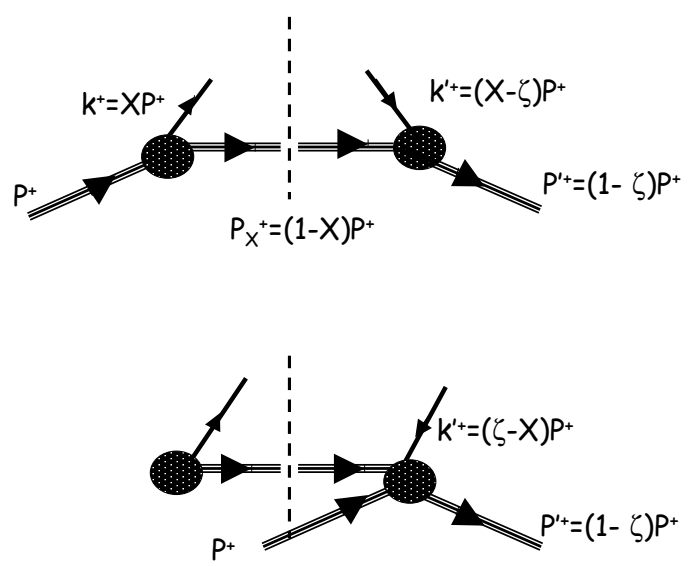

Fig. 2. Upper Panel: Parton Model; Lower Panel: Semi-disconnected contribution. The analytic structure of these diagrams was discussed for DIS [5].

that annihilates a quark with $X P^{+}$and creates an antiquark with $-(X-\zeta) P^{+}$. For the DGLAP region this cannot conserve plus momentum, but for the ERBL region, $-(X-\zeta) P^{+}$corresponds to an antiquark. Notice that the delta function has $P_{n}^{+}$ greater than $P^{+}$.

More heuristically, each of the matrix elements can, on one side describe the processes

$$
p \rightarrow q(q q) \quad \text { and } \quad q(q q) \rightarrow p
$$

respectively, at each vertex. The intermediate state $(q q)$ has the quantum numbers of a diquark with momentum $P_{n}$. This term has a clear partonic interpretation. On the other side, consistent with momentum conservation, one has two different types of intermediate states: i) $p \rightarrow q(q q q \bar{q})$ on the LHS, the $\bar{q}$ being re-emitted on the RHS; ii) $p \rightarrow q p(\bar{q})$ on the LHS, the $\bar{q}$ being re-emitted through the semidisconnected vertex of Fig.2b. In case $i$ ) the intermediate state has diquark quantum numbers, a partonic interpretation seems possible but with a catch that we explain in what follows. In case ii) the intermediate state is a $\bar{q}$. The semi-disconnected graphs do not correspond to a partonic description of the proton.

One can show when $X<\zeta$ that a crucial term in the matrix element corresponds to Fig. 2b, a "semi-disconnected" graph. In the DIS case, the identification of 
the initial and final matrix elements in Eq.(9) allows the replacement of the semidisconnected quark target diagrams for $X<0$ with the connected antiquark-target diagram for $X>0$ (with opposite sign). On the other hand for the GPD, because of the asymmetry between the initial quark-target state and the final state, the semidisconnected diagrams for $X-\zeta<0$ are equivalent to semi-disconnected diagrams for antiquark-target states, i.e. the ERBL region for quark-target amplitudes is the ERBL region for anti-quark target amplitudes.

Hence, there is a catch that casts a doubt on the possibility of giving a partonic interpretation of the ERBL region. By examining the analytic structure of GPDs, we know that in this region it is either one of the struck quarks/anti-quarks that is put on mass shell ${ }^{3}$, and not the state with diquark quantum numbers. The only way to have a $\bar{q}$ or equivalently a $q$, as an intermediate is by considering the diagram as in Fig. $2 \mathrm{~b}^{5}$, a semi-disconnected, non-partonic contribution. This development contradicts the partonic interpretation of the results for the ERBL region. In other words, the interpretations of the ERBL region, as would be obtained in Ref. 11, 13 by inserting different creation and annihilation operators does not address the issue of partonic interpretation.

We have a choice. We can take the contribution as $H_{X<\zeta}^{(2)}=0$. Alternatively we can conclude that there are non-partonic contributions to the GPDs and measurements of the ERBL region are not revealing the partonic content of the nucleons, or even the distribution of quark-antiquark meson states in the nucleon. We propose an approach to this in Ref. 14.

\section{Transverse Spin from Pseudoscalar Meson Production}

The basic definition of the quark-nucleon GPDs is through off-forward matrix elements of quark field correlators. Contracting with the Dirac matrices, $\gamma^{\mu}$ or $\gamma^{\mu} \gamma^{5}$ $\left(\sigma^{\mu \nu} \gamma^{5}\right)$, and integrating over the internal quark momenta gives rise to the four Chiral even (odd) GPDs, $H, E$ or $\widetilde{H}, \widetilde{E}$ (chiral odd: $H_{T}, E_{T}, \widetilde{H}_{T}, \widetilde{E}_{T}{ }^{7}$ ). The crucial connection of the 8 GPDs to spin dependent observables in DVCS and DVMP is through the helicity decomposition ${ }^{7}$, where, for example, one of the GPDs is given by chiral even helicity amplitudes obtained by substituting explicit Dirac helicity state spinors to yield

$$
H(x, \xi, t)=\frac{1}{\sqrt{1-\xi^{2}}}\left(A_{+,+;+,+}+A_{-,+;-,+}\right)-\frac{2 M \xi^{2}}{\Delta\left(1-\xi^{2}\right)}\left(A_{+,+;-,+}-A_{-,+;+,+}\right)
$$

We have constructed a robust model for the GPDs ${ }^{15}$, extending previous work ${ }^{16}$ that is based on the parameterization of diquark spectators and Regge behavior at small $X$. Our GPD model parameters are constrained by their relations to PDFs $($ at $\zeta=0, t=0) H^{q}(X, 0,0)=f_{1}^{q}(X), \tilde{H}^{q}(X, 0,0)=g_{1}^{q}(X), H_{T}^{q}(X, 0,0)=h_{1}^{q}(X)$ and to nucleon form factors $F_{1}(t), F_{2}(t), g_{A}(t), g_{P}(t)$ through the first $x$ moments. For Chiral odd GPDs there are fewer constraints. $H_{T}(X, 0,0)=h_{1}(X)$ can be fit 

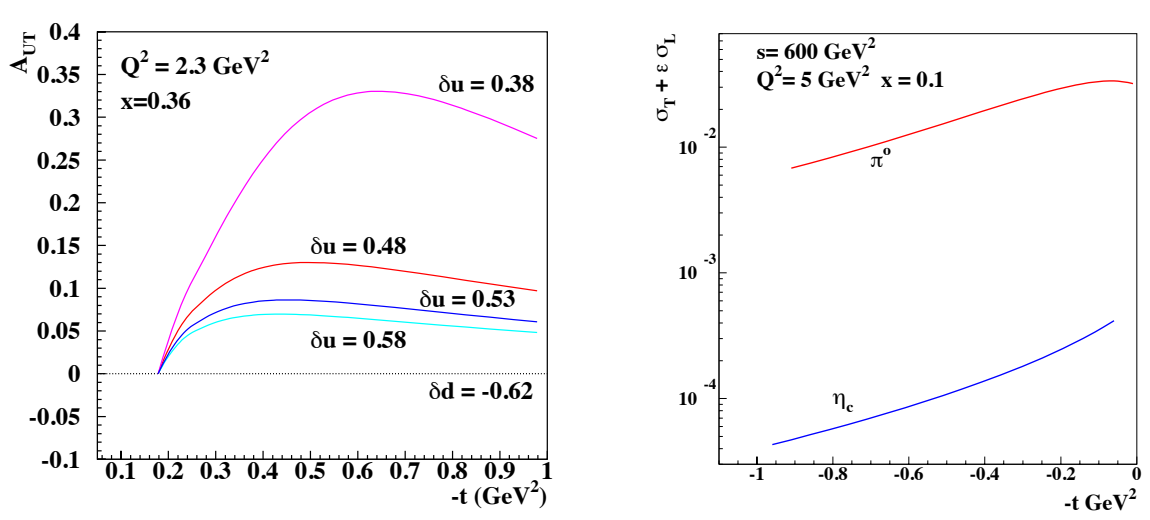

Fig. 3. Left: Transverse spin asymmetry, $A_{U T}$, vs. $-t$, at $Q^{2}=2.3 \mathrm{GeV}^{2}, x_{B j}=0.36$ for different values of the tensor charge, $\delta u$, with fixed $\delta d=-0.62$, i.e. equal to the central value extracted in a global fit ${ }^{17}$. Right: Comparison of $\pi^{o}$ and $\eta_{c}$ cross sections. Although $\eta_{c}$ is the hardest charmed meson to detect, the range between the two lines gives an estimate of where the cross sections for the other processes will lie.

to the loose constraints in Ref. 17 - the first moment of $H_{T}(X, \xi, t)$ is the "tensor form factor", called $g_{T}(t)$.

In the case of $\pi^{0}$ production there are important constraints that restrict the GPDs that contribute. Consider the $t$-channel quantum numbers corresponding to combinations of GPDs. The $x$ moments of the GPDs have expansions in terms of $t$ dependent form factors and polynomials in $\xi$. It has been shown by Lebed and Ji for pdfs $^{18}$ and Haegler for GPDs ${ }^{19}$ (see also Ref. 20), that these moments have $t$-channel angular momentum decompositions, as appropriate for $t$-channel exchanges, as well as Regge poles. From the $t$-channel perspective $\gamma^{*}+\pi^{0}$, has C-parity negative, and a restricted set of t-channel $J^{P C}$ values.

Consider first the chiral even GPDs that contribute to $\pi^{0}$. The crossing odd $\widetilde{H}$ has contributions from $2^{--}, 4^{--}$, etc. Although the crossing even, C-even, version has the boundary function $g_{1}(x)$, we expect that the odd case is suppressed. The crossing odd $\widetilde{E}$ has contributions from $1^{+-}, 2^{--}, 3^{+-}$, etc., so it is the leading candidate for chiral even GPDs that contribute to $\pi$. Its first moment is the pseudoscalar form factor, for which the main contribution is the $\pi$ itself. However, for the neutral $\pi$ this is not the case - there is no $\pi$ pole. That being said, in $\pi^{0}$ the non-pole contribution to the form factor is relevant, but undoubtedly small ${ }^{21}$.

The measured cross section for $\pi^{0}$ is sizable and has large transverse $\gamma^{*}$ contributions. This indicates that the main contributions should come from chiral odd GPDs, for which the $t$-channel decomposition is richer. We are thus led to the conclusion that chiral odd GPDs will dominate the neutral pseudoscalar leptoproduction cross sections. This result has interesting consequences. For one thing, in a factorized handbag picture, these GPDs will couple to the hard part, the 
$\gamma^{*}+$ quark $\rightarrow \pi^{0}+$ quark providing the $\pi^{0}$ couples through $\gamma^{5}$, which is naively twist 3, rather than the twist $2 \gamma^{+} \gamma^{5}$. Nevertheless, the previous arguments support this choice. Secondly the vector $1^{--}$and axial vector $1^{+-}$in the $t$-channel, viewed as particles $\left(\rho^{0}, \omega\right.$ and $\left.b_{1}^{0}, h\right)$, couple primarily to the transverse virtual photon. Guided by these observations ${ }^{22}$, we assume the hard part depends on whether the exchange quantum numbers are in the vector or axial vector series, thereby introducing orbital angular momentum into the model. We use $Q^{2}$ dependent electromagnetic "transition" form factors for vector or axial vector quantum numbers going to a pion. We calculate these using PQCD for $q+\bar{q}+\gamma^{*}\left(Q^{2}\right) \rightarrow q+\bar{q}$ and a standard $z$-dependent pion wave function, convoluted in an impact parameter representation that allows orbital excitations to be easily implemented.

With our model for the chiral odd, spin-dependent GPDs and these transition form factors, we can obtain the full range of cross sections and asymmetries in kinematic regimes that coincide with ongoing JLab experiments. (A similar emphasis on chiral odd contributions for $\pi$ electroproduction has recently been proposed ${ }^{23}$, although the details of that model are quite different from ours.) We are able to predict the important transverse photon contributions to the observables ${ }^{22}$. In figure 3 (left) we show one striking example of predictions that depend on the values of the tensor charges, thereby providing a means to narrow down those important quantities. This program has been presented ${ }^{15}$ and further details will soon appear, as the refinements of the chiral odd parameterization are completed ${ }^{24}$. In figure 3 (right) we show the cross section contribution, $\sigma_{T}+\epsilon \sigma_{L}$ for charmed meson production, as compared to $\pi^{0}$ production, based on a simple assumption about intrinsic charm content of the nucleon. This is a possibility to explore at future high energy lepton + nucleon accelerators.

\section{References}

1. I.V. Anikin and O.V. Teryaev, Phys. Rev. D76, 056007 (2007).

2. M. Diehl and D.Yu Ivanov, Eur. Phys. Jour. C 52, 919 (2007).

3. S. J. Brodsky and F. J. Llanes-Estrada, Eur. Phys. J. C 46, 751 (2006).

4. G.R. Goldstein and S. Liuti, Phys. Rev. D80, 071501 (2009).

5. R.L. Jaffe, Nucl. Phys. B229, 205 (1983); ibid Phys. Lett. 116B, 437 (1982).

6. A. V. Belitsky and A. V. Radyushkin, Phys. Rept. 418, 1 (2005)

7. M. Diehl, Phys. Rept. 388, 41 (2003).

8. J.C. Collins, T.C. Rogers and A.M. Stasto, Phys. Rev. D77, 085009 (2008).

9. S. J. Brodsky and F. J. Llanes-Estrada, A. P. Szczepaniak, arXiv:0812.0395 [hep-ph].

10. A. Accardi and J. W. Qiu, JHEP 0807, 090 (2008).

11. M. Diehl and T. Gousset, Phys. Lett. B 428, 359 (1998)

12. X. D. Ji, Phys. Rev. D 55, 7114 (1997).

13. K. J. Golec-Biernat and A. D. Martin, Phys. Rev. D 59, 014029 (1998)

14. G.R. Goldstein and S. Liuti, arXiv:1006.0213[hep-ph]

15. G.R. Goldstein, J.O. Gonzalez Hernandez, and S. Liuti, Phys. Rev. D84, 034007 (2011).

16. S. Ahmad, H. Honkanen, S. Liuti and S. K. Taneja, Phys. Rev. D 75, 094003 (2007); ibid EPJC (2009). 
17. M. Anselmino, M. Boglione, U. D'Alesio, A. Kotzinian, F. Murgia, A. Prokudin, C. Turk, Phys. Rev. D75, 054032 (2007). [hep-ph/0701006].

18. X. -D. Ji, R. F. Lebed, Phys. Rev. D63, 076005 (2001). [hep-ph/0012160].

19. P. .Hagler, Phys. Lett. B594, 164-170 (2004). [hep-ph/0404138].

20. M. Diehl, D. Y. .Ivanov, Eur. Phys. J. C52, 919-932 (2007). [arXiv:0707.0351 [hep$\mathrm{ph}]$.

21. K. Goeke, M. V. Polyakov, M. Vanderhaeghen, Prog. Part. Nucl. Phys. 47, 401-515 (2001). [hep-ph/0106012].

22. S. Ahmad, G. R. Goldstein, S. Liuti, Phys. Rev. D79, 054014 (2009). [arXiv:0805.3568 [hep-ph]].

23. S. V. Goloskokov, P. Kroll, Eur. Phys. J. C65, 137-151 (2010). [arXiv:0906.0460 [hep$\mathrm{ph}]$.

24. G.R. Goldstein and S. Liuti, in preparation. 